\title{
Genetic Algorithm Optimization for Determining Fuzzy Measures from Fuzzy Data
}

\author{
Chen Li, ${ }^{1,2}$ Gong Zeng-tai, ${ }^{1}$ and Duan Gang ${ }^{3}$ \\ ${ }^{1}$ College of Mathematics and Statistics, Northwest Normal University, Lanzhou 730070, China \\ ${ }^{2}$ Department of Mathematics, Lanzhou City University, Lanzhou 730070, China \\ ${ }^{3}$ School of Traffic and Transportation, Lanzhou Jiaotong University, Lanzhou 730070, China
}

Correspondence should be addressed to Gong Zeng-tai; zt-gong@163.com

Received 11 May 2013; Accepted 7 October 2013

Academic Editor: T. Warren Liao

Copyright (C) 2013 Chen Li et al. This is an open access article distributed under the Creative Commons Attribution License, which permits unrestricted use, distribution, and reproduction in any medium, provided the original work is properly cited.

\begin{abstract}
Fuzzy measures and fuzzy integrals have been successfully used in many real applications. How to determine fuzzy measures is a very difficult problem in these applications. Though there have existed some methodologies for solving this problem, such as genetic algorithms, gradient descent algorithms, neural networks, and particle swarm algorithm, it is hard to say which one is more appropriate and more feasible. Each method has its advantages. Most of the existed works can only deal with the data consisting of classic numbers which may arise limitations in practical applications. It is not reasonable to assume that all data are real data before we elicit them from practical data. Sometimes, fuzzy data may exist, such as in pharmacological, financial and sociological applications. Thus, we make an attempt to determine a more generalized type of general fuzzy measures from fuzzy data by means of genetic algorithms and Choquet integrals. In this paper, we make the first effort to define the $\sigma-\lambda$ rules. Furthermore we define and characterize the Choquet integrals of interval-valued functions and fuzzy-number-valued functions based on $\sigma-\lambda$ rules. In addition, we design a special genetic algorithm to determine a type of general fuzzy measures from fuzzy data.
\end{abstract}

\section{Introduction}

Fuzzy measures [1-4] and fuzzy integrals [5-9] have been applied successfully in multiattributes decision-making [10, 11], classification [12, 13], information fusion [14-18], nonlinear multiregression [19], feature selection [20, 21] and image processing. The reason of success is from the highly nonadditive and non-linear characteristics of fuzzy measures and fuzzy integrals. Fuzzy measure is the generalization of classical measure by using nonadditivity instead of additivity, which makes fuzzy measure be able to describe the importance of each individual information source (attribute or classifier) as well as the interaction [13], among them.

The Choquet integral [22-26] with respect to fuzzy measure is often used in information fusion and data mining as a nonlinear aggregation tool. The nonadditivity of fuzzy measures can effectively describe the interaction among the contributions from each attribute toward some target. Some works have shown successful applications of the Choquet integral in nonlinear multiregressions, classifications, and decisionmakings $[19,25,27-30]$, where the values of fuzzy measure are usually regarded as unknown parameter to be elicited from training data sets.

Most of existed works can only deal with the data consisting of classic numbers which may arise limitations in practical applications. It is not reasonable to assume that all data are real data before we elicit them from practical data. Sometimes, fuzzy data may exist, such as in pharmacological, financial, and sociological applications. Genetic algorithm (GA) is a stochastic search method for optimization problems based on the mechanics of natural selection and natural genetics. GA has demonstrated considerable success in providing good solutions to many complex optimization problems and received more and more attentions during the past three decades. The advantage of GA just make it able to obtain the global optimal solution fairly. In addition, compared with the traditional methods, GA has the ability to avoid getting stuck at a local optimal solution, since GA search from 
a single point. Thus, we make an attempt to determine a more generalized type of general fuzzy measures from fuzzy data by means of genetic algorithms and Choquet integrals.

The rest of this study is organized as follows. In Section 2, the basic definitions of fuzzy measures based on $\sigma-\lambda$ rules are reviewed. Section 3 briefly introduces the basic concepts on the Choquet integral of real-valued functions based on rules and gives the operational schemes of its on discrete sets. In Section 4, we formulate the problems to be solved. Section 5 uses genetic algorithm optimization to determine fuzzy measures from real-valued data. Section 6, introduces the Choquet integral of interval-valued functions based on rules. Consequently, we use genetic algorithm optimization to determine fuzzy measures from interval-valued data. Section 7 discusses the Choquet integral of fuzzy numbervalued functions based on rules, and then uses genetic algorithm optimization to determine fuzzy measures from fuzzy number-valued data. Finally, conclusions are made in Section 8 .

\section{Fuzzy Measure Based on $\sigma-\lambda$ Rules}

Definition 1. Let $X$ be a nonempty set and $\mathscr{A}$ a $\sigma$-algebra on the $X$. A set function $\mu$ is called a fuzzy measure based on $\sigma-\lambda$ rules if

$$
\mu\left(\bigcup_{i=1}^{\infty} A_{i}\right)= \begin{cases}\frac{1}{\lambda}\left\{\prod_{i=1}^{\infty}\left[1+\lambda \mu\left(A_{i}\right)\right]-1\right\}, & \lambda \neq 0, \\ \sum_{i=1}^{\infty} \mu\left(A_{i}\right), & \lambda=0,\end{cases}
$$

where $\lambda \in(-(1 / \sup \mu), \infty) \bigcup\{0\},\left\{A_{i}\right\} \subset \mathscr{A}$, and $A_{i} \cap A_{j}=\emptyset$ for all $i, j=1,2, \ldots$ and $i \neq j$.

Particularly, if $\lambda=0$, then $\sigma-\lambda$ rule is $\sigma$-additivity.

Definition 2. Let $\mathscr{A}$ be a $\sigma$-algebra on the $X$. $\mu$ is called Sugeno measure based on $\sigma-\lambda$ rules if $\mu$ satisfies $\sigma-\lambda$ rules and $\mu(X)=$ 1. Briefly we denoted $g_{\lambda}$.

Remark 3. In Definition 1 , if $n=2$, then

$$
\mu(A \cup B)= \begin{cases}\mu(A)+\mu(B)+\lambda \mu(A) \mu(B), & \lambda \neq 0, \\ \mu(A)+\mu(B), & \lambda=0 .\end{cases}
$$

Remark 4. In Definition $2, g_{\lambda}$ is a classical probability measure if $\lambda=0$, and it can be represented by a wide range of nonadditive measure as long as we select proper parameters, many scholars think that it is a very important kind of nonadditive measure [31-33].

Example 5. Three workers, $x_{1}, x_{2}$, and $x_{3}$, are engaged in producing the same kind of products; the efficiencies of every people are given as follows: $\mu\left(x_{1}\right)=5, \mu\left(x_{2}\right)=6$, and $\mu\left(x_{3}\right)=8$. Then we can get the joint efficiencies by use of $\sigma-\lambda$ rules as shown in Table 1 .

Remark 6. In Example 5, $x_{i}$ can be viewed as a attribute, $i=1,2,3$, we can calculate the contribution of their joint attributes by use of $\sigma-\lambda$ rules if we only know the contribution of individual attribute $g_{\lambda}\left(x_{i}\right), i=1,2,3$.
TABLE 1: The values of set function $\mu$ in Example 5 .

\begin{tabular}{lc}
\hline Set & Value of $\mu$ \\
\hline$E_{1}=\left\{x_{1}\right\}$ & 5 \\
$E_{2}=\left\{x_{2}\right\}$ & 6 \\
$E_{3}=\left\{x_{1}, x_{2}\right\}$ & $11+30 \lambda$ \\
$E_{4}=\left\{x_{3}\right\}$ & 8 \\
$E_{5}=\left\{x_{1}, x_{3}\right\}$ & $13+40 \lambda$ \\
$E_{6}=\left\{x_{2}, x_{3}\right\}$ & $14+48 \lambda$ \\
$E_{7}=\left\{x_{1}, x_{2}, x_{3}\right\}$ & $240 \lambda^{2}+118 \lambda+19$ \\
\hline
\end{tabular}

Theorem 7. Let $g_{\lambda}$ be a Sugeno measure based on $\sigma-\lambda$ rules. If $\lambda \geq 0$, then $g_{\lambda}$ has the monotonicity.

Proof. Let $E, F \in \mathscr{F}$ and $E \subset F$. Since $F=E \cup(F-E)$, this implies that

$$
\begin{gathered}
g_{\lambda}(F)=g_{\lambda}(E \cup(F-E))=g_{\lambda}(E) \\
+g_{\lambda}(F-E)+\lambda g_{\lambda}(E) g_{\lambda}(F-E), \\
g_{\lambda}(F) \geq g_{\lambda}(E)
\end{gathered}
$$

for $\lambda \geq 0$ and $g_{\lambda} \geq 0$.

Due to the limitation of the classical measure, Sugeno, the Japanese scholar, presents set functions called fuzzy measures which use the monotonicity instead of the additivity. In practical applications, we often use regular fuzzy measure [32] on finite sets.

Definition 8 (see [28]). Let $X$ be a finite set and $2^{X}$ be the power set of $X$. Set function $\mu: 2^{X} \rightarrow[0,1]$ is called a regular fuzzy measure defined on let $2^{X}$ if the following conditions are satisfied:

(1) $\mu(\emptyset)=0, \mu(X)=1$;

(2) if $E \in 2^{X}, G \in 2^{X}$, and $E \subset G$, then $\mu(E) \leq \mu(G)$.

Definition 9 (see [28]). Let $X$ be a finite set and $2^{X}$ be the power set, of $X$. A fuzzy measure $\mu: 2^{X} \rightarrow[0,1]$ is called a regular $\lambda$-fuzzy measure defined on let $2^{X}$ if the following conditions are satisfied:

(1) $\mu(\emptyset)=0, \mu(X)=1$;

(2) if $\mu(A \cup B)=\mu(A)+\mu(B)+\lambda \mu(A) \mu(B)$, where $A \subset$ $X, B \subset X, A \cap B=\emptyset$ and $\lambda \in(-1, \infty)$.

Theorem 10. Let $g_{\lambda}$ be a Sugeno measure based on $\sigma-\lambda$ rules. Then $g_{\lambda}$ is a regular $\lambda$-fuzzy measure defined on $\mathscr{A}$.

Proof. We could prove that $g_{\lambda}(\emptyset)=0$. Otherwise for every $\lambda \geq 0, A \in \mathscr{A}$, we have

$$
\begin{aligned}
g_{\lambda}(A) & =g_{\lambda}(A \cup \emptyset) \\
& =g_{\lambda}(A)+g_{\lambda}(\emptyset)+\lambda g_{\lambda}(A) g_{\lambda}(\emptyset), \\
g_{\lambda}(\emptyset) & =-\lambda g_{\lambda}(A) g_{\lambda}(\emptyset) .
\end{aligned}
$$


Since $g_{\lambda}(\emptyset) \neq 0$, we have $\lambda g_{\lambda}(\emptyset)=-1$; it is a contradiction. Furthermore, we obtain $g_{\lambda}(X)=1$, and $g_{\lambda}$ has the monotonicity by Definition 2 and Theorem 7 .

Denoting finite set $X=\left\{x_{1}, x_{2}, \ldots, x_{n}\right\}$, the value $g_{\lambda i}=$ $g_{\lambda}\left(x_{i}\right)$ for all $i=1,2, \ldots, n$ is called measure density.

Theorem 11. The parameter $\lambda$ of a regular Sugeno measure based on $\sigma-\lambda$ rules is determined by the following equation:

$$
\prod_{i=1}^{n}\left(1+\lambda g_{\lambda i}\right)=1+\lambda
$$

Proof. We can prove the above theorem by Theorem 10 and [32].

If we know the values of Sugeno measure based on $\sigma-\lambda$ on singleton sets, we can use Theorem 11 to obtain the values of $\lambda$ and then use Definition 1 to obtain the values on the other sets. It implies that a Sugeno measure based on $\sigma-\lambda$ can be determined by measure densities.

Theorem 12 (see [28]). If one knows the measure density $g_{\lambda i}$ on finite set $X=\left\{x_{1}, x_{2}, \ldots, x_{n}\right\}$, then there is only one solution $\lambda$ obtained from $\prod_{i=1}^{n}\left(1+\lambda g_{\lambda i}\right)=1+\lambda$.

\section{The Choquet Integrals of Real-Valued Function Based on $\sigma-\lambda$ Rules}

Definition 13 (see [34]). A regular fuzzy number, denoted by $\tilde{a}$, is a fuzzy subset of $\mathbb{R}$ with membership function $m: \mathbb{R} \rightarrow$ $[0,1]$ satisfying the following conditions.

(RFN1) there exists at least one number $a_{0} \in \mathbb{R}$ such that $a_{0}=1$;

(RFN2) $m(t)$ is nondecreasing on $\left(-\infty, a_{0}\right]$ and nonincreasing on $\left[a_{0},+\infty\right)$;

(RFN3) $m(t)$ is upper semicontinuous; that is, $\lim _{t \rightarrow t_{0}^{+}}=m\left(t_{0}\right)$ if $t_{0}<a_{0}$ and $\lim _{t \rightarrow t_{0}^{-}}=m\left(t_{0}\right)$ if $t_{0}>a_{0}$;

$$
\text { (RFN4) } \int_{-\infty}^{\infty} m(t) d t<\infty \text {. }
$$

The set of all regular fuzzy numbers is denoted by $\mathcal{N}$.

Let $f: X \rightarrow(-\infty, \infty)$ be a measurable function with respect to $\mathscr{A}$; that is, $f$ satisfies the condition that

$$
\{x \mid f(x) \geq \alpha\} \in \mathscr{A}
$$

for any $\alpha \in \mathbb{R}$.

From now on, we suppose that all functions defined on $X$ appearing as an integrand of the Choquet integral in this paper are measurable.

Definition 14 (see [22]). Let $(X, \mathscr{A})$ be a measurable space, and let $g_{\lambda}$ be a Sugeno measure based on $\sigma-\lambda$ rules on $\mathscr{A}$.
The Choquet integral of a real-valued function $f: X \rightarrow$ $(-\infty,+\infty)$ is defined as

$$
\begin{aligned}
\text { (c) } \int_{X} f d g_{\lambda}= & \int_{-\infty}^{0}\left[g_{\lambda}\left(F_{\alpha}\right)-g_{\lambda}(X)\right] d \alpha \\
& +\int_{0}^{\infty} g_{\lambda}\left(F_{\alpha}\right) d \alpha,
\end{aligned}
$$

where

$$
F_{\alpha}=\{x \mid f(x) \geq \alpha\}
$$

for $\alpha \in(-\infty,+\infty)$, if both of Riemann integrals exist and at least one of them has finite value.

Let $A \in \mathscr{A}$, then Choquet integral of a nonnegative realvalued function $f: X \rightarrow(0,+\infty)$ is defined as

$$
\text { (c) } \int_{A} f d g_{\lambda}=\int_{0}^{\infty} g_{\lambda}\left(A \cap F_{\alpha}\right) d \alpha \text {. }
$$

Without loss of the generality, Yang et al. [34] have proposed a new scheme to calculate the value of a Choquet integral with a real-valued integrand.

When $X=\left\{x_{1}, x_{2}, \ldots, x_{n}\right\}$, for any function $f: X \rightarrow$ $(-\infty,+\infty)$, both $\left[g_{\lambda}\left(F_{\alpha}\right)-g_{\lambda}(X)\right]$ and $g_{\lambda}\left(F_{\alpha}\right)$ are functions of $\alpha$ with bounded variance; therefore, their Riemann integrals with respect to $\alpha$ exist and are finite. So, Choquet integral (c) $\int f d g_{\lambda}$ is well defined. To calculate the value of the Choquet integral of a given real-valued function $f$, usually the values of $f$, that is, $f\left(x_{1}\right), f\left(x_{2}\right), \ldots, f\left(x_{n}\right)$, should be sorted in a nondecreasing order, so that $f\left(x_{1}^{\prime}\right) \leq f\left(x_{2}^{\prime}\right) \leq$ $\cdots \leq f\left(x_{n}^{\prime}\right)$, where $\left\{x_{1}^{\prime}, x_{2}^{\prime}, \ldots, x_{n}^{\prime}\right\}$ is a certain permutation of $\left\{x_{1}, x_{2}, \ldots, x_{n}\right\}$. Then, the value of the Choquet integral is obtained by

$$
\begin{aligned}
(c) \int f d g_{\lambda}=\sum_{i=1}^{n}[ & \left.f\left(x_{i}^{\prime}\right)-f\left(x_{i-1}^{\prime}\right)\right] \\
& \cdot g_{\lambda}\left(\left\{x_{i}^{\prime}, x_{i+1}^{\prime}, \ldots, x_{n}^{\prime}\right\}\right),
\end{aligned}
$$

where $f\left(x_{0}^{\prime}\right)=0$.

Example 15. Let $X=\left\{x_{1}, x_{2}, x_{3}\right\}, g_{\lambda}\left(x_{1}\right)=0.1, g_{\lambda}\left(x_{2}\right)=$ $0.2, g_{\lambda}\left(x_{3}\right)=0.3, f\left(x_{1}\right)=0.3, f\left(x_{2}\right)=0.7$, and $f\left(x_{3}\right)=$ 0.5 . We can obtain $\prod_{i=1}^{3}\left(1+\lambda g_{\lambda_{i}}\right)=1+\lambda$ by Theorem 11 . Furthermore we get $\lambda=3.1$.

By Definition 1, we can get the following results:

$$
\begin{aligned}
g_{\lambda}\left(\left\{x_{1}, x_{2}\right\}\right) & =g_{\lambda}\left(x_{1}\right)+g_{\lambda}\left(x_{2}\right)+\lambda g_{\lambda}\left(x_{1}\right) \cdot g_{\lambda}\left(x_{2}\right) \\
& =0.1+0.2+3.1 \times 0.1 \times 0.2 \\
& =0.362 .
\end{aligned}
$$

Similarly, $g_{\lambda}\left(\left\{x_{1}, x_{3}\right\}\right)=0.493, g_{\lambda}\left(\left\{x_{2}, x_{3}\right\}\right)=0.593, g_{\lambda}\left(\left\{x_{1}\right.\right.$, $\left.\left.x_{2}, x_{3}\right\}\right)=1$. 
TABLE 2: Data of Example 16.

\begin{tabular}{lccccc}
\hline$i$ & $f_{i 1}$ & $f_{i 2}$ & $f_{i 3}$ & $f_{i 4}$ & $f_{i 5}$ \\
\hline 1 & 1 & 0.6 & 0.8 & 0.8 & 1 \\
2 & 0.8 & 0.5 & 0.7 & 1 & 0.7 \\
3 & 0.8 & 0.8 & 0.5 & 0.8 & 0.9 \\
4 & 0.5 & 0.5 & 1 & 0.7 & 0.6 \\
5 & 0.2 & 0.9 & 0.7 & 0.6 & 0.3 \\
6 & 0.7 & 0.3 & 0.9 & 0.6 & 0.8 \\
7 & 1 & 0.6 & 1 & 0.5 & 0.8 \\
8 & 0.5 & 0.8 & 0.4 & 0.7 & 0.5 \\
9 & 0.4 & 0.7 & 0.6 & 0.9 & 0.2 \\
10 & 0.7 & 0.5 & 0.6 & 0.8 & 0.8 \\
\hline
\end{tabular}

By Definition 14, we can get the Choquet integrals of $f(x)$ with respect to $g_{\lambda}$ as follows:

$$
\begin{aligned}
\text { (c) } \int f d g_{\lambda}= & (0.3-0) \cdot 1+(0.5-0.3) \\
& \cdot g_{\lambda}\left(\left\{x_{3}, x_{2}\right\}\right)+(0.7-0.5) \\
& \cdot g_{\lambda}\left(x_{2}\right)=0.4586 .
\end{aligned}
$$

Example 16. Let $X=\left\{x_{1}, x_{2}, x_{3}, x_{4}, x_{5}\right\}, g_{\lambda}\left(x_{1}\right)=0.1$, $g_{\lambda}\left(x_{2}\right)=0.2, g_{\lambda}\left(x_{3}\right)=0.3, g_{\lambda}\left(x_{4}\right)=0.15$, and $g_{\lambda}\left(x_{5}\right)=$ 0.175 (Table 2).

By Theorem 11, we obtain that $\prod_{i=1}^{5}\left(1+\lambda g_{\lambda i}\right)=1+\lambda$, and with the aid of Mathematica software, we calculate that $\lambda=0.218$; furthermore, we get Choquet integrals which are shown in Table 3.

\section{Questions Description: Determine Fuzzy Measures}

In this section, we formulate our problems to be solved.

If we regard fuzzy integrals as multi-input single-output systems, we can obtain the Data through handling these systems. Suppose that we have several information sources $x_{1}, x_{2}, \ldots, x_{n}, n \geq 2$ and a given object $y$. Let $X=$ $\left\{x_{1}, x_{2}, \ldots, x_{n}\right\}$; we have the data with sample size $m$ as shown in Table 4 , where $f_{i j}$ is the $i$ th value of source $x_{j}$ and $E_{i}$ is the $i$ th value of object.

We hope to find a Sugeno measure $g_{\lambda}$ on measurable space $\left(X, 2^{X}\right)$, such that $E_{i}=(c) \int f_{i} d g_{\lambda}, i=1,2, \ldots, m$, where function $f_{i}$ is defined by $f_{i}\left(x_{j}\right)=f_{i j}, j=1,2, \ldots, n$ for $i=1,2, \ldots, m$.

If such a Sugeno measure $g_{\lambda}$ does not exist, we hope to find the optimally approximate solution. This is just the inverse problem of synthetic evaluation. We can also use the least square method to transform the above problem to a constrained optimization problem. An optimization problem is described as follows:

$$
\min (V)=\sqrt{\frac{1}{m} \sum_{i=1}^{m}\left(E_{i}-(c) \int f_{i} d g_{\lambda}\right)^{2}}
$$

A result of $\min (V)=0$ also means that a precise solution is found.

Here, we discuss this problem in three aspects. The first one is the values of $f_{i j}$, and $E_{i}(i=1,2, \ldots, m, j=1,2, \ldots, n)$ are a real-valued data. The second one is the values of $f_{i j}$, and $E_{i}(i=1,2, \ldots, m, j=1,2, \ldots, n)$ are an interval-valued data. The last one is $f_{i j}$, and $E_{i}(i=1,2, \ldots, m, j=1,2, \ldots, n)$ are a fuzzy-valued data.

\section{Using Genetic Algorithm to Determine Fuzzy Measures from Real-Valued Data}

In this section, we use genetic algorithm to determine fuzzy measures from real-valued data.

5.1. Genetic Algorithm (GA). Genetic algorithm (GA) is a stochastic search method for optimization problems based on the mechanics of natural selection and natural genetics (i.e., survival of the fittest). GA has demonstrated considerable success in providing good solutions to many complex optimization problems and received more and more attentions during the past three decades. When the objective functions to be optimized in the optimization problems are multimodal or the search spaces are particularly irregular, algorithms need to be highly robust in order to avoid getting stuck at a local optimal solution. The advantage of GA just makes it able to obtain the global optimal solution fairly. In addition, GA does not require the specific mathematical analysis of optimization problems, which makes GA easily coded by users who are not necessarily good at mathematics and algorithms.

5.1.1. The Decimal Coding. Chromosome $V$ is denoted by $V=$ $\left(a_{1}, a_{2}, \ldots, a_{n}\right)$, where gene $a_{i} \in[0,1]$ for all $i=1,2, \ldots, n$, and $a_{1}=g_{\lambda 1}, a_{2}=g_{\lambda 2}, \ldots$, and $a_{n-1}=g_{\lambda n-1}, a_{n}=\lambda$.

5.1.2. The Decoding. Find the formula

$$
g_{\lambda n}=\frac{1}{\lambda}\left(\frac{1+\lambda}{\prod_{i=1}^{n-1}\left(1+\lambda g_{\lambda i}\right)}-1\right)
$$

by $g_{\lambda i}(i=1,2, \ldots, n-1), \lambda$, and Definition 1 . Furthermore, the values of $g_{\lambda}\left(x_{1}, x_{2}, \ldots, x_{k}\right)$ for all $k=1,2, \ldots, n$ can be obtained by Definition 1 . The encoding can guarantee to get a feasible solution. That is, the solution satisfies Definition 1, and it will not undermine the feasibility of the solution no matter what kind of genetic operation (crossover or mutation) be used to chromosome.

5.1.3. The Arithmetic Crossover. Use the crossover probability $P_{c}$ to choose two parent chromosomes $V^{1}=\left(a_{1}^{1}, a_{2}^{1}, \ldots, a_{n}^{1}\right)$ 
TABLE 3: Results of Example 16.

\begin{tabular}{lcccc}
\hline$(c) \int f_{1} d g_{\lambda}$ & $(c) \int f_{2} d g_{\lambda}$ & $(c) \int f_{3} d g_{\lambda}$ & $(c) \int f_{4} d g_{\lambda}$ & $(c) \int f_{5} d g_{\lambda}$ \\
\hline 0.8 & 0.7 & 0.71 & 0.79 & 0.58 \\
\hline$(c) \int f_{6} d g_{\lambda}$ & $(c) \int f_{7} d g_{\lambda}$ & $(c) \int f_{8} d g_{\lambda}$ & $(c) \int f_{9} d g_{\lambda}$ & $(c) \int f_{10} d g_{\lambda}$ \\
\hline 0.67 & 0.79 & 0.56 & 0.56 & 0.65 \\
\hline
\end{tabular}

TABle 4: Data.

\begin{tabular}{lcccc}
\hline$x_{1}$ & $x_{2}$ & $\cdots$ & $x_{n}$ & $y$ \\
\hline$f_{11}$ & $f_{12}$ & $\cdots$ & $f_{1 n}$ & $E_{1}$ \\
$f_{21}$ & $f_{22}$ & $\cdots$ & $f_{2 n}$ & $E_{2}$ \\
$\vdots$ & $\vdots$ & $\vdots$ & $\vdots$ & $\vdots$ \\
$f_{m 1}$ & $f_{m 2}$ & $\cdots$ & $f_{m n}$ & $E_{m}$ \\
\hline
\end{tabular}

and $V^{2}=\left(a_{1}^{2}, a_{2}^{2}, \ldots, a_{n}^{2}\right)$ and use the arithmetic crossover to get two offspring chromosomes $V^{3}$ and $V^{4}$ :

$$
\begin{aligned}
V^{3}=\alpha V^{1}+(1-\alpha) V^{2}= & \left(\alpha a_{1}^{1}+(1-\alpha) a_{1}^{2}, \alpha a_{2}^{1}\right. \\
& \left.+(1-\alpha) a_{2}^{2}, \ldots, \alpha a_{n}^{1}+(1-\alpha) a_{n}^{2}\right), \\
V^{4}=(1-\alpha) V^{1}+\alpha V^{2}=( & (1-\alpha) a_{1}^{1}+\alpha a_{1}^{2},(1-\alpha) a_{2}^{1} \\
& \left.+\alpha a_{2}^{2}, \ldots,(1-\alpha) a_{n}^{1}+\alpha a_{n}^{2}\right),
\end{aligned}
$$

where $\alpha \in[0,1]$.

5.1.4. The Nonuniform Mutation. Select parent chromosome $V=\left(a_{1}, a_{2}, \ldots, a_{n}\right)$ according to the mutation probability $P_{m}$ and take the mutation to $a_{k}$ for the random generation of $k$ in $[1, n]$. Let

$$
a_{k}^{\prime}=a_{k}+\phi\left(t, a_{k}^{U}-a_{k}\right)
$$

or

$$
a_{k}^{\prime}=a_{k}-\phi\left(t, a_{k}-a_{k}^{L}\right),
$$

where $a_{k}^{U}$ and $a_{k}^{L}$ are the upper and lower bound of $a_{k}$, respectively, and $t$ is the number of generations. Function $\phi(t, b)$ is defined as follows:

$$
\phi(t, b)=b \cdot r \cdot\left(1-\frac{t}{T}\right)^{b}
$$

where $r$ is a random number of $[0,1], T$ is the largest number of generations, and $b$ is the parameter. Obviously, $\lim _{n \rightarrow T} \phi(t, b)=0$.

\subsubsection{The Evaluation Function. Evaluation function is defined} by

$$
\min (V)=\sqrt{\frac{1}{n} \sum_{i=1}^{n}\left(E_{i}-(c) \int f_{i} d g_{\lambda}\right)^{2}}
$$

where $(c) \int f_{i} d g_{\lambda}$ is defined by Definition 14 . Use the objective function as the evaluation function of a single chromosome.

The genetic algorithm procedure is summarized as follows.

Step 1. Initialize pop size chromosomes randomly.

Step 2. Update the chromosomes by crossover and mutation operations.

Step 3. Calculate the evaluation function for all chromosomes.

Step 4. Select the chromosomes by spinning the roulette wheel.

Step 5. Repeat the Step 2 to Step 3 for a given number of cycles.

Step 6. Report the best chromosome as the optimal solution.

\subsection{Examples and Results}

Example 17. A railway administration chooses 15 passengers randomly to evaluate the passenger train plan in the administration (Table 5). Customer bases its overall scores on transfer times, in-train congestion, travel time, and ticket price, and also they have a score for each of four aspects. Let $X=$ $\left\{x_{1}, x_{2}, x_{3}, x_{4},\right\}$ be the attributes of transfer times, congestion, travel time, and ticket price, respectively. We want to know which attribute is the most important for passengers. Here, $P_{c}=0.85, P_{m}=0.3$, and the population size is 20 .

We can obtain that the transfer times are the most important for passengers from Table 6 and the convergence rate of Example 17 as shown in Figure 1.

\section{Using Genetic Algorithm to Determine Fuzzy Measures from Interval-Valued Data}

The intervals are derived from many practical application problems, when instead of knowing the precise values $x$ of some quantity $X$ we know only the intervals $[\underline{x}, \bar{x}]$, in which $X$, ranges. Since the comparison of two values or quantities is the basic and most frequently used step in optimization, interval-valued function plays an important role in interval computation development.

6.1. The Choquet Integrals of Interval-Valued Function Based on $\sigma-\lambda$ Rules. With the definitions of the preceding subsections and from $\mathrm{Wu}$ et al. [35], we assume that $R^{+}=[0,+\infty)$, 
TABLE 5: Data of Example 17 (the customer evaluation).

\begin{tabular}{|c|c|c|c|c|c|}
\hline Customer & Transfer times & Congestion & Travel time & Ticket price & Evaluation $E_{i}$ \\
\hline 1 & 0 & 0 & 0.3 & 1 & 0.2 \\
\hline 2 & 0.3 & 0.8 & 0.5 & 0.6 & 0.5 \\
\hline 3 & 0.7 & 0.9 & 1 & 0.7 & 0.8 \\
\hline 4 & 0.1 & 0 & 0.4 & 0.3 & 0.15 \\
\hline 5 & 0.5 & 0.4 & 0.5 & 0.6 & 0.5 \\
\hline 6 & 1 & 0.3 & 0.8 & 0.8 & 0.7 \\
\hline 7 & 0.3 & 1 & 0 & 1 & 0.5 \\
\hline 8 & 0.9 & 0.6 & 0.5 & 0.7 & 0.7 \\
\hline 9 & 0.6 & 0.8 & 0.2 & 0.4 & 0.5 \\
\hline 10 & 0.4 & 1 & 0.5 & 0 & 0.4 \\
\hline 11 & 0.8 & 0.4 & 1 & 0.3 & 0.6 \\
\hline 12 & 1 & 0.8 & 0.7 & 0.5 & 0.75 \\
\hline 13 & 0.2 & 0.6 & 1 & 0.7 & 0.5 \\
\hline 14 & 0 & 0.3 & 0.2 & 0.9 & 0.2 \\
\hline 15 & 0.4 & 0.2 & 0.8 & 0 & 0.3 \\
\hline
\end{tabular}

TABLE 6: Results of Example 17.

\begin{tabular}{lcccc}
\hline Set & $\lambda$ & $g_{\lambda}$ & Error & $\begin{array}{c}\text { Number of } \\
\text { generation }\end{array}$ \\
\hline$\left\{x_{1}\right\}$ & & 0.2969 & & \\
$\left\{x_{2}\right\}$ & 0.965 & 0.1842 & 0.0154 & 100 \\
$\left\{x_{3}\right\}$ & & 0.1347 & & \\
$\left\{x_{4}\right\}$ & & 0.1529 & & \\
\hline
\end{tabular}

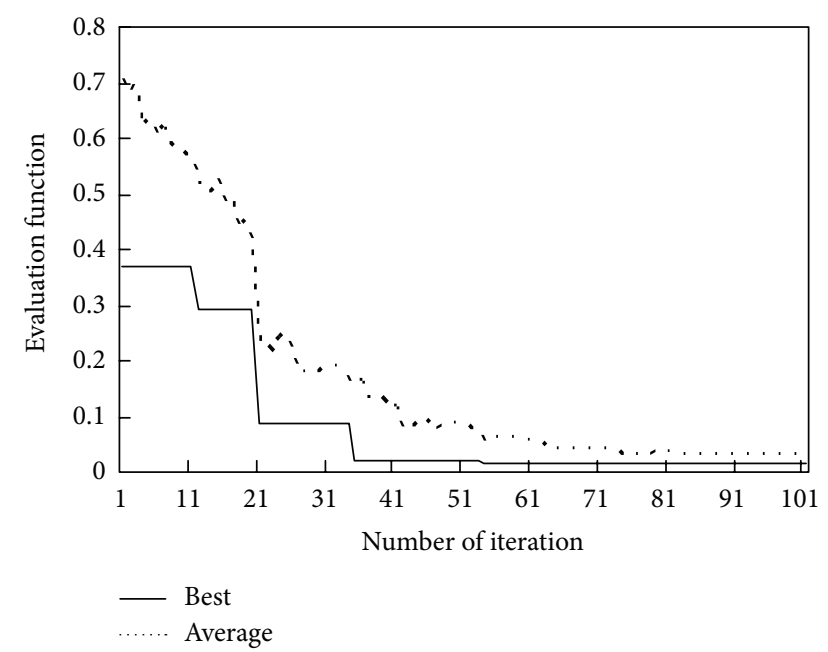

FIGURE 1: The convergence rate of Example 17.

$I\left(R^{+}\right)=\left\{\bar{r}:\left[r^{-}, r^{+}\right] \subset R^{+}\right\}$is the set of interval numbers, and $\bar{F}(X)$ is the set of all interval numbers $\bar{f}$.

Interval numbers satisfy the following basic operations:

(1) $\bar{r} * \bar{p}=\left[r^{-} * p^{-}, r^{+} * p^{+}\right](*+\mathrm{\vee} \wedge)$;

(2) $k \cdot \bar{r}=\left[k r^{-}, k r^{+}\right],\left(k \in \mathbb{R}^{+}\right)$;

(3) $\bar{r} \leq \bar{p} \Leftrightarrow r^{-} \leq p^{-}, r^{+} \leq p^{+}$;
(4) $d(\bar{r}, \bar{p})=\max \left\{\left|r^{-}-p^{-}\right|,\left|r^{+}-p^{+}\right|\right\}$;

(5) if $d\left(\bar{r}_{n}, \bar{r}\right) \rightarrow 0$, then $\bar{r}_{n} \rightarrow \bar{r}$.

Definition 18 (see [34]). An interval-valued function $\bar{f}$ : $X \rightarrow I\left(R^{+}\right)$is measurable if both $f^{-}(x)$ and $f^{+}(x)$ are measurable function of $x$, where $\bar{f}(x)=\left[f^{-}(x), f^{+}(x)\right]$, $f^{-}(x)$ is the left end point of interval $\bar{f}(x)$, and $f^{+}(x)$ is the right end point of interval $\bar{f}(x)$.

Definition 19. Let $\left(X, \mathscr{A}, g_{\lambda}\right)$ be a nonadditive measure space based on $\sigma$ - $\lambda$ rules $\bar{f}: X \rightarrow I\left(R^{+}\right)$a measurable function in $X$ and $E \in \mathscr{A}$. Then the Choquet integral of $\bar{f}$ with respect to $g_{\lambda}$ is defined by

$$
\text { (c) } \int_{E} \bar{f} d g_{\lambda}=:\left\{(c) \int_{E} \bar{f} d g_{\lambda} \mid g \in S_{\bar{f}(x)}\right\}
$$

if $(c) \int_{E} \bar{f} d g_{\lambda}$ is a closed interval on $I\left(R^{+}\right)$, where $S_{\bar{f}(x)}=\{g \mid$ $\left.g: X \rightarrow R^{+}\right\}$is a measurable selection on $\bar{f}(x)$.

Theorem 20. Let $\bar{f}: X \rightarrow I\left(R^{+}\right)$be a measurable intervalvalued function on $X$, and let $g_{\lambda}$ be a Sugeno measure based on $\sigma-\lambda$ rules on $\mathscr{A}$. The Choquet integral of $\bar{f}$ with respect to $g_{\lambda}$ is

$$
\text { (c) } \int \bar{f} d g_{\lambda}=\left[(c) \int f^{-} d g_{\lambda},(c) \int f^{+} d g_{\lambda}\right] \text {, }
$$

where $f^{-}(x)$ is the left end point of interval $\bar{f}(x)$ and $f^{+}(x)$ is the right end point of interval $\bar{f}(x)$, for every $x \in X$.

Proof. We can prove the above theorem by Theorem 10 and [34].

Using the continuity and the monotonicity of the Choquet integral with the nonnegativity and the monotonicity of the fuzzy measures, we may obtain the following theorem.

Theorem 21. Let $\bar{f}: X \rightarrow I\left(R^{+}\right)$be a measurable intervalvalued function on $X$, let $g_{\lambda}$ be a sugeno measure based on 
$\sigma-\lambda$ rules on $\mathscr{A}$, and $X=\left\{x_{1}, x_{2}, \ldots, x_{n}\right\}$. Then, the value of Choquet integral of $\bar{f}$ with respect to $g_{\lambda}$ is obtained by

$$
\begin{aligned}
(c) \int \bar{f} d g_{\lambda} & \\
= & {\left[\sum_{i=1}^{n}\left[f^{-}\left(x_{i}^{\prime}\right)-f^{-}\left(x_{i-1}^{\prime}\right)\right]\right.} \\
& \cdot g_{\lambda}\left(\left\{x_{i}^{\prime}, x_{i+1}^{\prime}, \ldots, x_{n}^{\prime}\right\}\right), \\
& \sum_{i=1}^{n}\left[f^{+}\left(x_{i}^{\prime \prime}\right)-f^{+}\left(x_{i-1}^{\prime \prime}\right)\right] \\
& \left.\cdot g_{\lambda}\left(\left\{x_{i}^{\prime \prime}, x_{i+1}^{\prime \prime}, \ldots, x_{n}^{\prime \prime}\right\}\right)\right],
\end{aligned}
$$

where $f^{-}\left(x_{0}^{\prime}\right)=0, f^{+}\left(x_{0}^{\prime \prime}\right)=0$, the values of $f^{-}$and $f^{+}$, that is, $f^{-}\left(x_{1}\right), f^{-}\left(x_{2}\right), \ldots, f^{-}\left(x_{n}\right)$, and $f^{+}\left(x_{1}\right), f^{+}\left(x_{2}\right), \ldots, f^{+}\left(x_{n}\right)$ should be sorted in a nondecreasing order, so that $f^{-}\left(x_{1}^{\prime}\right) \leq$ $f^{-}\left(x_{2}^{\prime}\right) \leq \cdots \leq f^{-}\left(x_{n}^{\prime}\right)$ and $f^{+}\left(x_{1}^{\prime \prime}\right) \leq f^{+}\left(x_{2}^{\prime \prime}\right) \leq \cdots \leq f^{+}\left(x_{n}^{\prime \prime}\right)$, respectively, and $\left\{x_{1}^{\prime}, x_{2}^{\prime}, \ldots, x_{n}^{\prime}\right\}$ and $\left\{x_{1}^{\prime \prime}, x_{2}^{\prime \prime}, \ldots, x_{n}^{\prime \prime}\right\}$ are a certain permutation of $\left\{x_{1}, x_{2}, \ldots, x_{n}\right\}$, respectively.

6.2. Using Genetic Algorithm to Determine Fuzzy Measures from Interval-Valued Data. In this subsection, we use genetic algorithm to determine fuzzy measures from interval-valued data.

6.2.1. Genetic Algorithm (GA). In this subsection, the all algorithms are the same to the Section 5.1 but the evaluation function. Here, the valuation function is defined by

$$
\begin{aligned}
\operatorname{Eval}(V)=\left(\frac { 1 } { m } \sum _ { i = 1 } ^ { m } \operatorname { m i n } \left\{\left((c) \int f_{i}^{-} d g_{\lambda}-E_{i}^{-}\right)^{2},\right.\right. & \\
& \left.\left.\left((c) \int f_{i}^{+} d g_{\lambda}-E_{i}^{+}\right)^{2}\right\}\right)^{1 / 2},
\end{aligned}
$$

where (c) $\int f_{i}^{-} d g_{\lambda}$ and (c) $\int f_{i}^{+} d g_{\lambda}$ are defined by Definition 14 and Theorem 20.

\subsubsection{Examples and Results}

Example 22. If the evaluation information in Example 17 is represented by the interval-valued fuzzy numbers as shown in Table 7, then we redetermine fuzzy measures from intervalvalued data by using genetic optimization.

We can obtain that the transfer times are the most important for passengers from Table 8 and the convergence rate of Example 22 as shown in Figure 2.

\section{Using Genetic Algorithm to Determine Fuzzy Measures from Fuzzy-Valued Data}

In many respects, fuzzy numbers depict the physical world more realistically than single-valued numbers. suppose, for

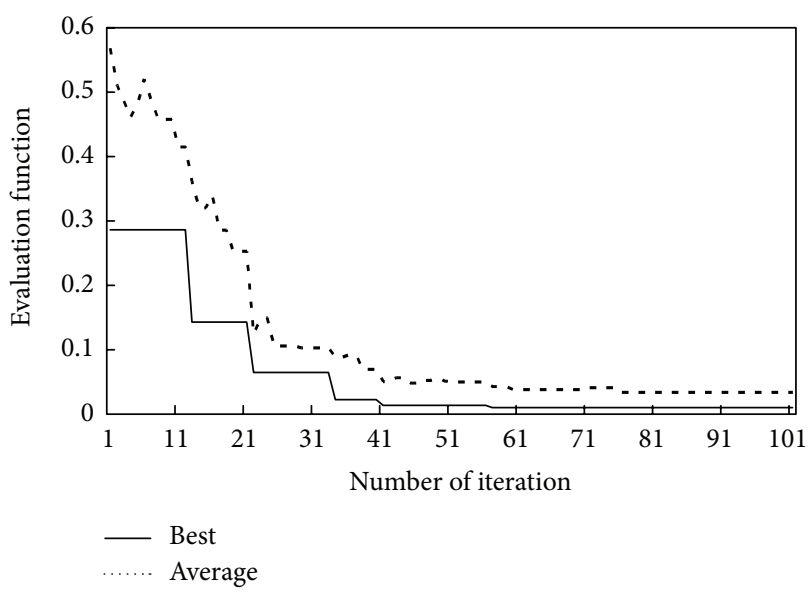

FIgURE 2: The convergence rate of Example 22.

example, service quality is an intangible asset of enterprises that is related to customers judgments about the overall quality of a firm. Fuzzy numbers are used in statistics, computer programming, engineering (especially communications), and experimental science. The concept takes into account the fact that all phenomena in the physical universe have a degree of inherent uncertainty.

Definition 23 (see $[33,36,37]$ ). Let $(X, \widetilde{F})$ be a fuzzy measurable space, and let $\mathscr{F}^{*}(\mathbb{R})$ be the class of all fuzzy subsets of $\mathbb{R}$. A fuzzy-valued function $\tilde{f}: X \rightarrow \mathscr{F}^{*}(\mathbb{R})$ is called a measurable function if for every $\lambda \in(0,1]$, its $\alpha$-cut $f_{\alpha}(x)$ is measurable, where

$$
f_{\alpha}(x)=(\tilde{f}(x))_{\alpha}=\{r \mid \tilde{f}(x)(r) \geq \alpha\} .
$$

Remark 24. A measurable fuzzy-valued function is a especially measurable fuzzy set-value function.

Let $\tilde{f}: X \rightarrow \widetilde{\mathscr{F}}, \forall \lambda \in(0,1]$, and $(\widetilde{f}(x))_{\alpha}=$ $\left[(\tilde{f}(x))_{\alpha}^{-},(\tilde{f}(x))_{\alpha}^{+}\right]$. We will simply denote that

$$
f_{\alpha}^{-}(x)=(\tilde{f}(x))_{\alpha}^{-}, f_{\alpha}^{+}(x)=(\tilde{f}(x))_{\alpha}^{+} .
$$

Obviously, $f_{\alpha}^{-}(x)$ and $f_{\alpha}^{+}(x)$ are real functions.

From now on, we suppose that all functions defined on $X$ appearing as an integrand of the Choquet integral in this paper are measurable.

According to Theorem $10, g_{\lambda}$ is a signed fuzzy measure on $\mathscr{A}$. Therefore, we may give the following definition referring to [34].

Fuzzy-valued function $\tilde{f}: X \rightarrow \widetilde{F}$ is said to be a $C$-integrally bounded, if there exists a Choquet integrable function $h: X \rightarrow R^{+}$such that $|\dot{x}| \leq h(t)$ for $\dot{x} \in[\tilde{f}(t)]_{0}$.

Definition 25. Let $\tilde{f}: X \rightarrow \widetilde{F}$ be a measurable fuzzy-valued function on $X$, and let $g_{\lambda}$ be a Sugeno measure based on $\sigma$ $\lambda$ rules. Assume that $\widetilde{f}$ is $C$-integrally bounded. $\tilde{f}$ is called Choquet integrable with respect to $g_{\lambda}$ if

$$
\left\{\left[(c) \int \tilde{f} \mathrm{~d} \mu\right]_{\lambda}=:\left\{(c) \int g \mathrm{~d} \mu \mid g \in S_{f_{\lambda}}\right\}, 0 \leq \lambda \leq 1\right\}
$$


TABLE 7: Data of Example 22 (the customer evaluation).

\begin{tabular}{|c|c|c|c|c|c|}
\hline Customer & Transfer times & Congestion & Travel time & Ticket price & Evaluation $E_{i}$ \\
\hline 1 & {$[0.00,0.00]$} & {$[0.00,0.00]$} & {$[0.25,0.35]$} & {$[1.00,1.00]$} & {$[0.15,0.25]$} \\
\hline 2 & {$[0.25,0.35]$} & {$[0.15,0.25]$} & {$[0.45,0.55]$} & {$[0.55,0.65]$} & {$[0.45,0.55]$} \\
\hline 3 & {$[0.65,0.75]$} & {$[0.85,0.95]$} & {$[1.00,1.00]$} & {$[0.65,0.75]$} & {$[0.75,0.85]$} \\
\hline 4 & {$[0.05,0.15]$} & {$[0.00,0.00]$} & {$[0.35,0.45]$} & {$[0.25,0.35]$} & {$[0.10,0.20]$} \\
\hline 5 & {$[0.45,0.55]$} & {$[0.35,0.45]$} & {$[0.45,0.55]$} & {$[0.55,0.65]$} & {$[0.45,0.55]$} \\
\hline 6 & {$[1.00,1.00]$} & {$[0.25,0.35]$} & {$[0.75,0.85]$} & {$[0.75,0.85]$} & {$[0.65,0.75]$} \\
\hline 7 & {$[0.25,0.35]$} & {$[1.00,1.00]$} & {$[0.00,0.00]$} & {$[1.00,1.00]$} & {$[0.45,0.55]$} \\
\hline 8 & {$[0.85,0.95]$} & {$[0.55,0.65]$} & {$[0.45,0.55]$} & {$[0.65,0.75]$} & {$[0.65,0.75]$} \\
\hline 9 & {$[0.55,0.65]$} & {$[0.75,0.85]$} & {$[0.15,0.25]$} & {$[0.35,0.45]$} & {$[0.45,0.55]$} \\
\hline 10 & {$[0.35,0.45]$} & {$[1.00,1.00]$} & {$[0.45,0.55]$} & {$[0.00,0.00]$} & {$[0.35,0.45]$} \\
\hline 11 & {$[0.75,0.85]$} & {$[0.35,0.45]$} & {$[1.00,1.00]$} & {$[0.25,0.35]$} & {$[0.55,0.65]$} \\
\hline 12 & {$[1.00,1.00]$} & {$[0.75,0.85]$} & {$[0.65,0.75]$} & {$[0.45,0.55]$} & {$[0.70,0.80]$} \\
\hline 13 & {$[0.15,0.25]$} & {$[0.55,0.65]$} & {$[1.00,1.00]$} & {$[0.65,0.75]$} & {$[0.45,0.55]$} \\
\hline 14 & {$[0.00,0.00]$} & {$[0.25,0.35]$} & {$[0.15,0.25]$} & {$[0.85,0.95]$} & {$[0.15,0.25]$} \\
\hline 15 & {$[0.35,0.45]$} & {$[0.15,0.25]$} & {$[0.75,0.85]$} & {$[0.00,0.00]$} & {$[0.25,0.35]$} \\
\hline
\end{tabular}

TABLE 8: Results of Example 22.

\begin{tabular}{lcccc}
\hline Set & $\lambda$ & $g_{\lambda}$ & Error & $\begin{array}{c}\text { Number of } \\
\text { generation }\end{array}$ \\
\hline$\left\{x_{1}\right\}$ & & 0.3186 & & \\
$\left\{x_{2}\right\}$ & 0.937 & 0.1819 & 0.010 & 100 \\
$\left\{x_{3}\right\}$ & & 0.1407 & & \\
$\left\{x_{4}\right\}$ & & 0.1344 & & \\
\hline
\end{tabular}

confirms a unique fuzzy number $\widetilde{a} \in \widetilde{\mathscr{F}}$, which is denoted by $(c) \int \widetilde{f} \mathrm{~d} \mu=\tilde{a}$, where $S_{f_{\lambda}}=\left\{g: X \rightarrow R, g \in f_{\lambda}\right.$ is a measurable selection of $\left.f_{\lambda}\right\}$.

The exact membership function of the Choquet integral with respect to Sugeno fuzzy measure $g_{\lambda}$ for fuzzy-valued integrand is rather difficult to be found. In a simpler but common case where $X=\left\{x_{1}, x_{2}, \ldots, x_{n}\right\}$ is finite, according to Definition 25, the calculation of the Choquet integral with a fuzzy-valued function comes down to that of the Choquet integral with an interval-valued function. Here, let us look at examples to show how to calculate the fuzzy-valued Choquet integral with respect to a Sugeno measure $g_{\lambda}$.

Example 26. Let $\widetilde{f}\left(x_{1}\right)=[0,1,1], \widetilde{f}\left(x_{2}\right)=[0.5,0.5,1.5]$, and $\widetilde{f}\left(x_{3}\right)=[0,1,2]$. Here, a triangular fuzzy number is denoted by $\left[a_{l}, a_{0}, a_{r}\right]$, where $a_{l}, a_{0}, a_{r} \in \mathbb{R}$ and $a_{l} \leq a_{0} \leq a_{r}$. Set function $g_{\lambda}$ is a sugeno measure based on $\sigma-\lambda$ rules. Function $\tilde{f}$ is triangular fuzzy valued. The membership function of $\widetilde{f}\left(x_{1}\right), \widetilde{f}\left(x_{2}\right)$, and $\widetilde{f}\left(x_{3}\right)$ are

$$
\begin{gathered}
m_{1}(t)=m_{\tilde{f}\left(x_{1}\right)}(t)= \begin{cases}t, & t \in[0,1], \\
0, & t \notin[0,1],\end{cases} \\
m_{2}(t)=m_{\tilde{f}\left(x_{2}\right)}(t)= \begin{cases}1.5-t, & t \in[0.5,1.5], \\
0, & t \notin[0.5,1.5],\end{cases}
\end{gathered}
$$

$$
m_{3}(t)=m_{\tilde{f}\left(x_{3}\right)}(t)= \begin{cases}t, & t \in[0,1] \\ 2-t, & t \in(1,2] \\ 0, & t \notin[0,2]\end{cases}
$$

respectively. They are shown in Figure 1. The $\alpha$-cut of $\tilde{f}$ is represented by interval

$$
\begin{aligned}
\tilde{f}_{\alpha}\left(x_{1}\right) & =M_{\alpha}^{\tilde{f}\left(x_{1}\right)}=\left\{t \mid m_{\tilde{f}\left(x_{1}\right)}(t) \geq \alpha\right\}=[\alpha, 1] ; \\
\tilde{f}_{\alpha}\left(x_{2}\right) & =M_{\alpha}^{\tilde{f}\left(x_{2}\right)}=\left\{t \mid m_{\tilde{f}\left(x_{2}\right)}(t) \geq \alpha\right\} \\
& =[0.5,1.5-\alpha] ; \\
\tilde{f}_{\alpha}\left(x_{3}\right) & =M_{\alpha}^{\tilde{f}\left(x_{3}\right)}=\left\{t \mid m_{\tilde{f}\left(x_{3}\right)}(t) \geq \alpha\right\} \\
& =[\alpha, 2-\alpha], \quad \text { where } \alpha \in[0,1] .
\end{aligned}
$$

It is easy to get $\left[\tilde{f}_{\alpha}\left(x_{1}\right)\right]_{l}=\alpha,\left[\tilde{f}_{\alpha}\left(x_{2}\right)\right]_{l}=0.5,\left[\tilde{f}_{\alpha}\left(x_{3}\right)\right]_{l}=$ $\alpha,\left[\tilde{f}_{\alpha}\left(x_{1}\right)\right]_{r}=1,\left[\tilde{f}_{\alpha}\left(x_{2}\right)\right]_{r}=1.5-\alpha$, and $\left[\tilde{f}_{\alpha}\left(x_{3}\right)\right]_{r}=2-\alpha$.

We conclude that

(1) When $0 \leq \alpha \leq 0.5$, we get $\left.\left[\tilde{f}_{\alpha}\left(x_{1}\right)\right]_{l}=\tilde{f}_{\alpha}\left(x_{3}\right)\right]_{l} \leq$ $\left[\tilde{f}_{\alpha}\left(x_{2}\right)\right]_{l}$. Using Definition 14, we can let $x_{1}^{\prime}=x_{1}, x_{2}^{\prime}=$ $x_{3}, x_{3}^{\prime}=x_{2}$, and then

$$
\begin{aligned}
& {\left[(c) \int \tilde{f}_{\alpha} d g_{\lambda}\right]_{l}=}\left\{\left[f\left(x_{1}^{\prime}\right)\right]_{l}-\left[f\left(x_{0}^{\prime}\right)\right]_{l}\right\} \\
& \cdot g_{\lambda}\left(x_{1}^{\prime}, x_{2}^{\prime}, x_{3}^{\prime}\right)+\left\{\left[f\left(x_{2}^{\prime}\right)\right]_{l}-\left[f\left(x_{1}^{\prime}\right)\right]_{l}\right\} \\
& g_{\lambda}\left(x_{2}^{\prime}, x_{3}^{\prime}\right)+\left\{\left[f\left(x_{3}^{\prime}\right)\right]_{l}-\left[f\left(x_{2}^{\prime}\right)\right]_{l}\right\} \cdot g_{\lambda}\left(x_{3}^{\prime}\right) \\
&=[\alpha-0] \cdot 1+[\alpha-\alpha] \cdot 0.5931+[0.5-\alpha] \cdot 0.3 \\
&=0.7 \alpha+0.15
\end{aligned}
$$

where $\left[f\left(x_{0}^{\prime}\right)\right]_{l}=0,\left[f\left(x_{0}^{\prime}\right)\right]_{r}=0$. 
TABLE 9: Data of Example 27 (The customer evaluation).

\begin{tabular}{lccccc}
\hline Customer & Transfer times & Congestion & Travel time & Ticket price & Evaluation $E_{i}$ \\
\hline 1 & {$[-0.1,0.00,0.10]$} & {$[-0.1,0.00,0.10]$} & {$[0.20,0.30,0.40]$} & {$[0.90,1.00,1.10]$} & {$[0.10,0.20,0.30]$} \\
2 & {$[0.20,0.30,0.40]$} & {$[0.70,0.80,0.90]$} & {$[0.40,0.50,0.60]$} & {$[0.50,0.60,0.70]$} & {$[0.40,0.50,0.60]$} \\
3 & {$[0.60,0.70,0.80]$} & {$[0.80,0.90,1.00]$} & {$[0.90,1.00,1.10]$} & {$[0.60,0.70,0.80]$} & {$[0.70,0.80,0.90]$} \\
4 & {$[0.00,0.10,0.20]$} & {$[-0.1,0.00,0.10]$} & {$[0.30,0.40,0.50]$} & {$[0.20,0.30,0.40]$} & {$[0.05,0.15,0.25]$} \\
5 & {$[0.40,0.50,0.60]$} & {$[0.30,0.40,0.50]$} & {$[0.40,0.50,0.60]$} & {$[0.50,0.60,0.70]$} & {$[0.40,0.50,0.60]$} \\
\hline
\end{tabular}

TABLE 10: The triangular fuzzy number changed into the interval number.

\begin{tabular}{lccc}
\hline Customer & Transfer times & Congestion & Travel time \\
\hline 1 & {$[0.1 \alpha-0.1,-0.1 \alpha+0.1]$} & {$[0.1 \alpha-0.1,-0.1 \alpha+0.1]$} & {$[0.1 \alpha+0.2,-0.1 \alpha+0.4]$} \\
2 & {$[0.1 \alpha+0.2,-0.1 \alpha+0.4]$} & {$[0.1 \alpha+0.7,-0.1 \alpha+0.9]$} & {$[0.1 \alpha+0.4,-0.1 \alpha+0.6]$} \\
3 & {$[0.1 \alpha+0.6,-0.1 \alpha+0.8]$} & {$[0.1 \alpha+0.8,-0.1 \alpha+1.0]$} & {$[0.1 \alpha+0.9,-0.1 \alpha+1.1]$} \\
4 & {$[0.1 \alpha,-0.1 \alpha+0.2]$} & {$[0.1 \alpha-0.1,-0.1 \alpha+0.1]$} & {$[0.1 \alpha+0.3,-0.1 \alpha+0.5]$} \\
5 & {$[0.1 \alpha+0.4,-0.1 \alpha+0.6]$} & {$[0.1 \alpha+0.3,-0.1 \alpha+0.5]$} & {$[0.1 \alpha+0.4,-0.1 \alpha+0.6]$} \\
\hline Customer & Ticket price & Evaluation $E_{i}$ & \\
\hline 1 & {$[0.1 \alpha+0.9,-0.1 \alpha+1.1]$} & {$[0.1 \alpha+0.1,-0.1 \alpha+0.3]$} & \\
2 & {$[0.1 \alpha+0.5,-0.1 \alpha+0.7]$} & {$[0.1 \alpha+0.4,-0.1 \alpha+0.6]$} & \\
3 & {$[0.1 \alpha+0.6,-0.1 \alpha+0.8]$} & {$[0.1 \alpha+0.7,-0.1 \alpha+0.9]$} & \\
4 & {$[0.1 \alpha+0.2,-0.1 \alpha+0.4]$} & {$[0.1 \alpha+0.05,-0.1 \alpha+0.25]$} & \\
5 & {$[0.1 \alpha+0.5,-0.1 \alpha+0.7]$} & {$[0.1 \alpha+0.4,-0.1 \alpha+0.6]$} & \\
\hline
\end{tabular}

TABLE 11: Results of Example 27.

\begin{tabular}{|c|c|c|c|c|}
\hline Set & $\lambda$ & $g_{\lambda}$ & Error & $\begin{array}{l}\text { Number of } \\
\text { generation }\end{array}$ \\
\hline$\left\{x_{1}\right\}$ & \multirow{4}{*}{0.05} & 0.4292 & \multirow{4}{*}{0.0049} & \multirow{4}{*}{100} \\
\hline$\left\{x_{2}\right\}$ & & 0.2428 & & \\
\hline$\left\{x_{3}\right\}$ & & 0.1586 & & \\
\hline$\left\{x_{4}\right\}$ & & 0.1524 & & \\
\hline
\end{tabular}

When $0 \leq \alpha \leq 0.5$, we get $\left[\tilde{f}_{\alpha}\left(x_{1}\right)\right]_{r} \leq\left[\tilde{f}_{\alpha}\left(x_{2}\right)\right]_{r} \leq$ $\left[\tilde{f}_{\alpha}\left(x_{3}\right)\right]_{r}$. Using Definition 14 we can let $x_{1}^{\prime}=x_{1}, x_{2}^{\prime}=$ $x_{2}$, and $x_{3}^{\prime}=x_{3}$, and then

$$
\begin{aligned}
& {\left[(c) \int \tilde{f}_{\alpha} d g_{\lambda}\right]_{r}=}\left\{\left[f\left(x_{1}^{\prime}\right)\right]_{r}-\left[f\left(x_{0}^{\prime}\right)\right]_{r}\right\} \\
& \cdot g_{\lambda}\left(x_{1}^{\prime}, x_{2}^{\prime}, x_{3}^{\prime}\right) \\
&+\left\{\left[f\left(x_{2}^{\prime}\right)\right]_{r}-\left[f\left(x_{1}^{\prime}\right)\right]_{r}\right\}, \\
& g_{\lambda}\left(x_{2}^{\prime}, x_{3}^{\prime}\right)+\left\{\left[f\left(x_{3}^{\prime}\right)\right]_{r}-\left[f\left(x_{2}^{\prime}\right)\right]_{r}\right\} \cdot g_{\lambda}\left(x_{3}^{\prime}\right) \\
&=[1-0] \cdot 1+[1.5-\alpha-1] \\
& \cdot 0.593+[2-\alpha-1.5+\alpha] \cdot 0.3 \\
&=1.4465-0.593 \alpha .
\end{aligned}
$$

That is, $(c) \int \tilde{f}_{\alpha} d g_{\lambda}=[0.7 \alpha+0.15,1.45-0.592]$.
(2) When $0.5<\alpha \leq 1$, we get $\left[\tilde{f}_{\alpha}\left(x_{2}\right)\right]_{l}<\left[\tilde{f}_{\alpha}\left(x_{3}\right)\right]_{l}=$ $\left[\tilde{f}_{\alpha}\left(x_{1}\right)\right]_{l}$. Using Definition 14, we can let $x_{1}^{\prime}=x_{2}, x_{2}^{\prime}=$ $x_{1}$, and $x_{3}^{\prime}=x_{3}$, and then

$$
\begin{aligned}
& {\left[(c) \int \tilde{f}_{\alpha} d g_{\lambda}\right]_{l}=}\left\{\left[f\left(x_{1}^{\prime}\right)\right]_{l}-\left[f\left(x_{0}^{\prime}\right)\right]_{l}\right\} \\
& \cdot g_{\lambda}\left(x_{1}^{\prime}, x_{2}^{\prime}, x_{3}^{\prime}\right) \\
&+\left\{\left[f\left(x_{2}^{\prime}\right)\right]_{l}-\left[f\left(x_{1}^{\prime}\right)\right]_{l}\right\}, \\
& g_{\lambda}\left(x_{2}^{\prime}, x_{3}^{\prime}\right)+\left\{\left[f\left(x_{3}^{\prime}\right)\right]_{l}-\left[f\left(x_{2}^{\prime}\right)\right]_{l}\right\} \cdot g_{\lambda}\left(x_{3}^{\prime}\right) \\
&=0.5 \cdot 1+[\alpha-\alpha] \cdot 0.3+[\alpha-0.5] \cdot 0.493 \\
&=0.493 \alpha+0.2535,
\end{aligned}
$$

where $\left[f\left(x_{0}^{\prime}\right)\right]_{l}=0,\left[f\left(x_{0}^{\prime}\right)\right]_{r}=0$.

When $0.5<\alpha \leq 1$, we get $\left[\tilde{f}_{\alpha}\left(x_{2}\right)\right]_{r} \leq\left[\tilde{f}_{\alpha}\left(x_{1}\right)\right]_{r} \leq$ $\left[\tilde{f}_{\alpha}\left(x_{3}\right)\right]_{r}$. Using Definition 14, we can let $x_{1}^{\prime}=x_{2}, x_{2}^{\prime}=$ $x_{1}$, and $x_{3}^{\prime}=x_{3}$, and then

$$
\begin{aligned}
{\left[(c) \int \tilde{f}_{\alpha} d g_{\lambda}\right]_{r}=} & \left\{\left[f\left(x_{1}^{\prime}\right)\right]_{r}-\left[f\left(x_{0}^{\prime}\right)\right]_{r}\right\} \\
& \cdot g_{\lambda}\left(x_{1}^{\prime}, x_{2}^{\prime}, x_{3}^{\prime}\right) \\
& +\left\{\left[f\left(x_{2}^{\prime}\right)\right]_{r}-\left[f\left(x_{1}^{\prime}\right)\right]_{r}\right\},
\end{aligned}
$$




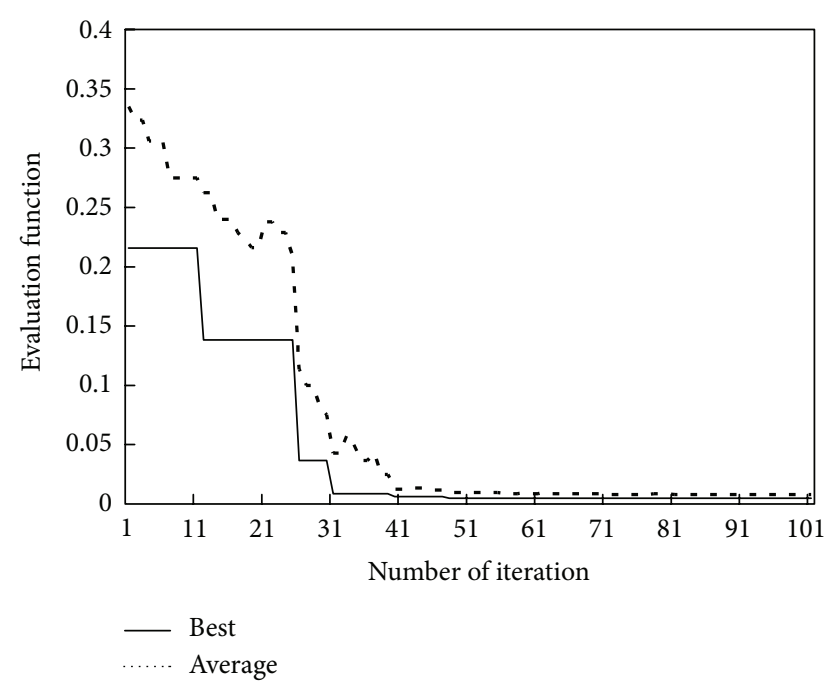

FIGURE 3: The convergence rate of Example 27.

$$
\begin{aligned}
g_{\lambda}\left(x_{2}^{\prime}, x_{3}^{\prime}\right)+\left\{\left[f\left(x_{3}^{\prime}\right)\right]_{r}-\left[f\left(x_{2}^{\prime}\right)\right]_{r}\right\} \cdot g_{\lambda}\left(x_{3}^{\prime}\right) \\
=[1.5-\alpha] \cdot 1+[1-(1.5-\alpha)] \\
\cdot 0.4931+[(2-\alpha)-1] \cdot 0.3 \\
=1.55-0.8 \alpha .
\end{aligned}
$$

That is, $(c) \int \widetilde{f}_{\alpha} d g_{\lambda}=[0.493 \alpha+0.2535,1.55-0.8 \alpha]$.

Example 27. If the evaluation information in Example 17 is represented by the triangular fuzzy number as shown in Table 9, then we redetermine fuzzy measures from fuzzyvalued data by using genetic algorithm.

Fist, we use the method as shown in Example 26 to change the triangular fuzzy number of Table 9 into interval number as shown in Table 10; that is, the optimization of triangular fuzzy number comes down to that of the interval number.

We can obtain that the transfer times are the most important for passengers from Table 11 and the convergence rate of Example 27 as shown in Figure 3, which represents the convergence rate of Example 27, where the solid line represents the convergence rate of the target value about the best optimal solution, and dotted line represents the convergence rate of average value of the target value about all the solutions.

\section{Conclusions and Remarks}

In this paper, we have considered the genetic algorithm optimization for determining fuzzy measures from fuzzy data. We have gotten joint measures by use of single measure with the aid of $\sigma-\lambda$ rules. Then we have formulated our problems to be solved; that is, how to determine the fuzzy measures from fixed data. Furthermore, we have introduced the Choquet integral of interval-valued functions and then given the genetic algorithm optimization to determine fuzzy measures from interval-valued data. Finally we have discussed the Choquet integral of fuzzy number-valued functions. Consequently, we have given the genetic algorithm optimization to determine fuzzy measures from fuzzy number-valued data.

\section{Acknowledgments}

The authors would like to thank the referees and editors for providing very helpful comments and suggestions. This work is supported by the Natural Scientific Funds of China (71061013, 61262022), the Scientific Research Project of Northwest Normal University (no. NWNU-KJCXGC-03-61), and Lanzhou Jiaotong University, Young Scientific Research Fund Project (2011020).

\section{References}

[1] H.-C. Liu, Y.-D. Jheng, W.-C. Lin, and G.-S. Chen, "A novel fuzzy measure and its choquet integral regression model," in Proceedings of the 6th International Conference on Machine Learning and Cybernetics (ICMLC '07), pp. 1394-1398, August 2007.

[2] M. A. Mohamed and W. Xiao, "Q-measures: an efficient extension of the Sugeno $\lambda$-measure," IEEE Transactions on Fuzzy Systems, vol. 11, no. 3, pp. 419-426, 2003.

[3] P. Miranda and M. Grabisch, "P-symmetric fuzzy measures," in Proceedings of the 9th International Conference of Information Processing and Management of Uncertainty in Knowledge-Based Systems, pp. 545-552, Annecy, France, 2002.

[4] R. R. Yager, "Uncertainty representation using fuzzy measures," IEEE Transactions on Systems, Man, and Cybernetics B, vol. 32, no. 1, pp. 13-20, 2002.

[5] M. Friedman, M. Ming, and A. Kandel, "On typical values and fuzzy integrals," IEEE Transactions on Systems, Man, and Cybernetics B, vol. 27, no. 4, pp. 703-705, 1997.

[6] Z. Wang, K.-S. Leung, M.-L. Wong, and J. Fang, "A new type of nonlinear integrals and the computational algorithm," Fuzzy Sets and Systems, vol. 112, no. 2, pp. 223-231, 2000.

[7] Z. Wang, K. Xu, P.-A. Heng, and K.-S. Leung, "Indeterminate integrals with respect to nonadditive measures," Fuzzy Sets and Systems, vol. 138, no. 3, pp. 485-495, 2003.

[8] Z. Xu, "Choquet integrals of weighted intuitionistic fuzzy information," Information Sciences, vol. 180, no. 5, pp. 726-736, 2010.

[9] Y. Ouyang, R. Mesiar, and H. Agahi, "An inequality related to Minkowski type for Sugeno integrals," Information Sciences, vol. 180, no. 14, pp. 2793-2801, 2010.

[10] X.-Z. Wang, J.-H. Zhai, and S.-X. Lu, "Induction of multiple fuzzy decision trees based on rough set technique," Information Sciences, vol. 178, no. 16, pp. 3188-3202, 2008.

[11] X.-Z. Wang, S.-F. Zhang, and J.-H. Zhai, "A nonlinear integral defined on partition and its application to decision trees," Soft Computing, vol. 11, no. 4, pp. 317-321, 2007.

[12] X.-Z. Wang, S.-X. Lu, and J.-H. Zhai, "Fast fuzzy multicategory SVM based on support vector domain description," International Journal of Pattern Recognition and Artificial Intelligence, vol. 22, no. 1, pp. 109-120, 2008. 
[13] R. Yang, Z. Wang, P.-A. Heng, and K.-S. Leung, "Classification of heterogeneous fuzzy data by choquet integral with fuzzyvalued integrand," IEEE Transactions on Fuzzy Systems, vol. 15, no. 5, pp. 931-942, 2007.

[14] B. Biggio, G. Fumera, and F. Roli, "Multiple classifier systems for robust classifier design in adversarial environments," International Journal of Machine Learning and Cybernetics, vol. 1, no. 1-4, pp. 27-41, 2010.

[15] G. Büyüközkan and D. Ruan, "Choquet integral based aggregation approach to software development risk assessment," Information Sciences, vol. 180, no. 3, pp. 441-451, 2010.

[16] S.-B. Cho and J. H. Kim, "Combining multiple neural networks by fuzzy integral for robust classification," IEEE Transactions on Systems, Man and Cybernetics, vol. 25, no. 2, pp. 380-384, 1995.

[17] H. Tahani and J. M. Keller, "Information fusion in computer vision using the fuzzy integral," IEEE Transactions on Systems, Man and Cybernetics, vol. 20, no. 3, pp. 733-741, 1990.

[18] L. J. Wang, "An improved multiple fuzzy NNC system based on mutual information and fuzzy integral," International Journal of Machine Learning and Cybernetics, vol. 2, no. 1, pp. 25-36, 2011.

[19] K.-S. Leung, M.-L. Wong, W. Lam, Z. Wang, and K. Xu, "Learning nonlinear multiregression networks based on evolutionary computation," IEEE Transactions on Systems, Man, and Cybernetics B, vol. 32, no. 5, pp. 630-644, 2002.

[20] Q. Hu, W. Pan, S. An, P. Ma, and J. Wei, "An efficient gene selection technique for cancer recognition based on neighborhood mutual information," International Journal of Machine Learning and Cybernetics, vol. 1, no. 1-4, pp. 63-74, 2010.

[21] D. L. Tong and R. Mintram, "Genetic Algorithm-Neural Network (GANN): a study of neural network activation functions and depth of genetic algorithm search applied to feature selection," International Journal of Machine Learning and Cybernetics, vol. 1, no. 1-4, pp. 75-87, 2010.

[22] G. Choquet, “Theory of capacities," Annales de l'Institut Fourier, vol. 5, pp. 131-295, 1954.

[23] D. Denneberg, Non-Additive Measure and Integral, vol. 27, Kluwer Academic, Boston, Mass, USA, 1994.

[24] T. Murofushi, M. Sugeno, and M. Machida, "Non-monotonic fuzzy measures and the Choquet integral," Fuzzy Sets and Systems, vol. 64, no. 1, pp. 73-86, 1994.

[25] Z. Wang, K.-S. Leung, M.-L. Wong, and J. Fang, "A new type of nonlinear integrals and the computational algorithm," Fuzzy Sets and Systems, vol. 112, no. 2, pp. 223-231, 2000.

[26] K. Xu, Z. Wang, P.-A. Heng, and K.-S. Leung, "Classification by nonlinear integral projections," IEEE Transactions on Fuzzy Systems, vol. 11, no. 2, pp. 187-201, 2003.

[27] M. Grabisch, "New algorithm for identifying fuzzy measures and its application to pattern recognition," in Proceedings of the IEEE International Conference on Fuzzy Systems (IFES '95), pp. 145-150, Yokohama, Japan, March 1995.

[28] X.-Z. Wang, Y.-L. He, L.-C. Dong, and H.-Y. Zhao, "Particle swarm optimization for determining fuzzy measures from data," Information Sciences, vol. 181, no. 19, pp. 4230-4252, 2011.

[29] Z. Wang, G. J. Klir, and W. Wang, "Monotone set functions defined by Choquet integral," Fuzzy Sets and Systems, vol. 81, no. 2, pp. 241-250, 1996.

[30] K. Xu, Z. Wang, M.-L. Wong, and K.-S. Leung, "Discover dependency pattern among attributes by using a new type of nonlinear multiregression," International Journal of Intelligent Systems, vol. 16, no. 8, pp. 949-962, 2001.
[31] M. H. Ha, Y. Li, J. Li, and D. Z. Tian, "Key theorem of learning theory and uniform convergence velocity boundary in Sugeno measure space," China Science E, vol. 36, no. 4, pp. 398-410, 2006 (Chinese).

[32] Z. Y. Wang and G. J. Klir, Fuzzy Measure Theory, Plenum Press, New York, NY, USA, 1992.

[33] S. Weber, "Two integrals and some modified versions-critical remarks," Fuzzy Sets and Systems, vol. 20, no. 1, pp. 97-105, 1986.

[34] R. Yang, Z. Wang, P.-A. Heng, and K.-S. Leung, "Fuzzy numbers and fuzzification of the Choquet integral," Fuzzy Sets and Systems, vol. 153, no. 1, pp. 95-113, 2005.

[35] C. Wu, D. Zhang, C. Guo, and C. Wu, "Fuzzy number fuzzy measures and fuzzy integrals (1)," Fuzzy Sets and Systems, vol. 98, no. 3, pp. 355-360, 1998.

[36] M. H. Ha and C. X. Wu, The Theory of Fuzzy Measure and Fuzzy Integral, Science Press, Beijing, China, 1998, Chinese.

[37] C. Wu and Z. Gong, "On Henstock integrals of interval-valued functions and fuzzy-valued functions," Fuzzy Sets and Systems, vol. 115, no. 3, pp. 377-391, 2000. 


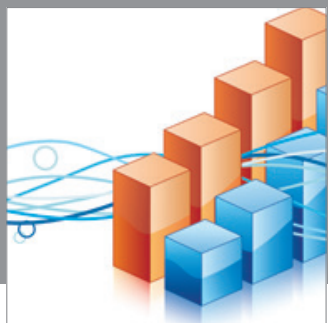

Advances in

Operations Research

mansans

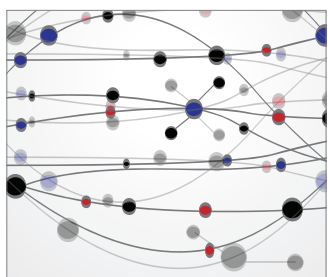

The Scientific World Journal
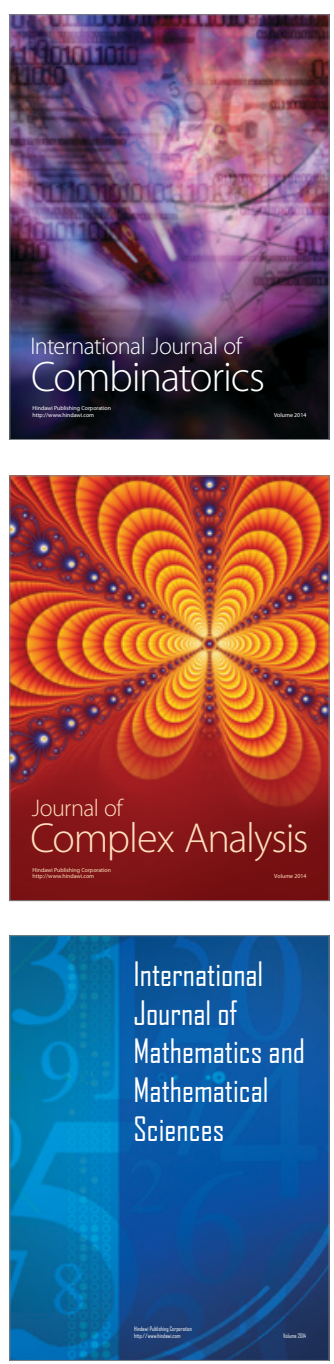
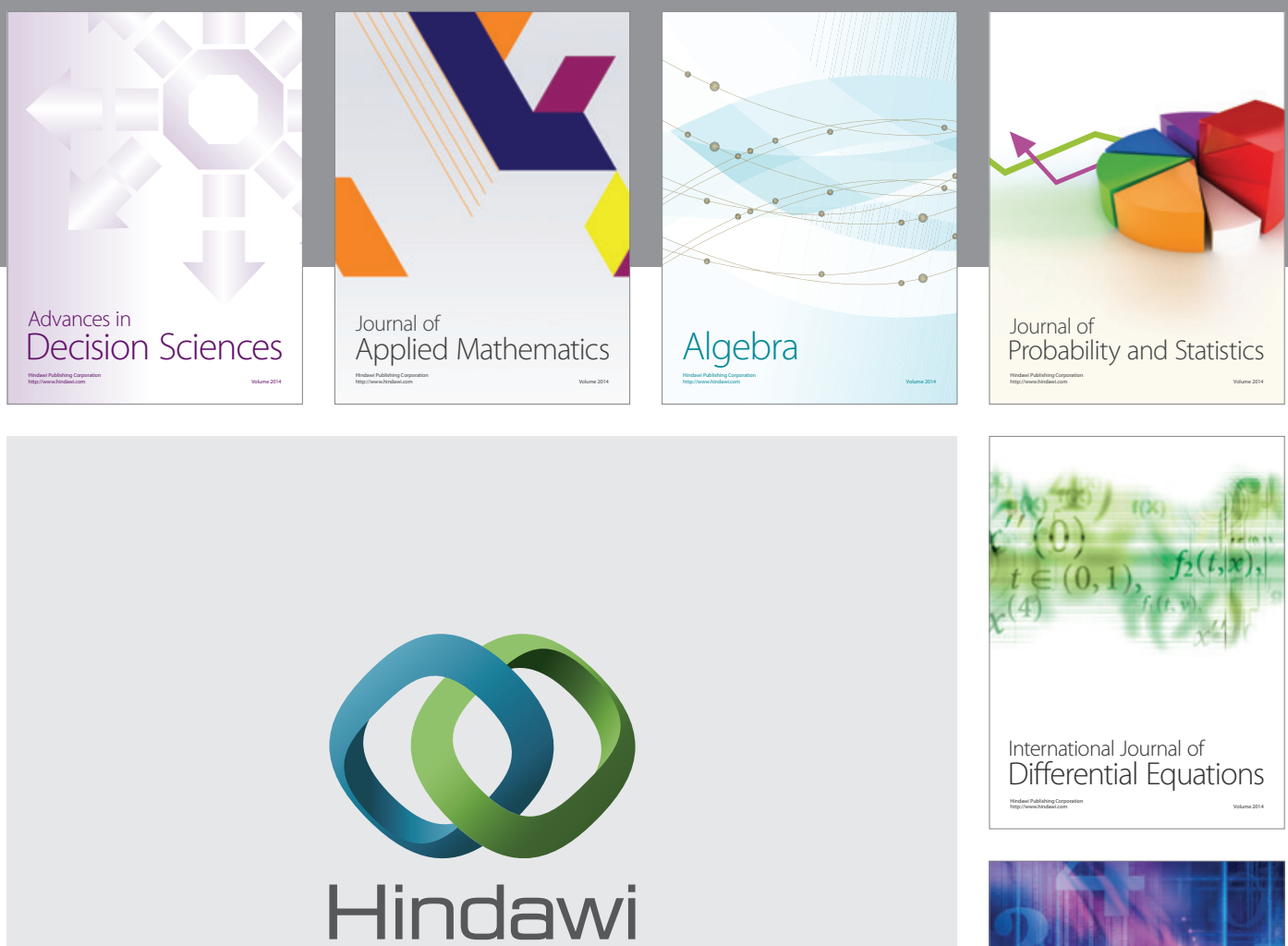

Submit your manuscripts at http://www.hindawi.com
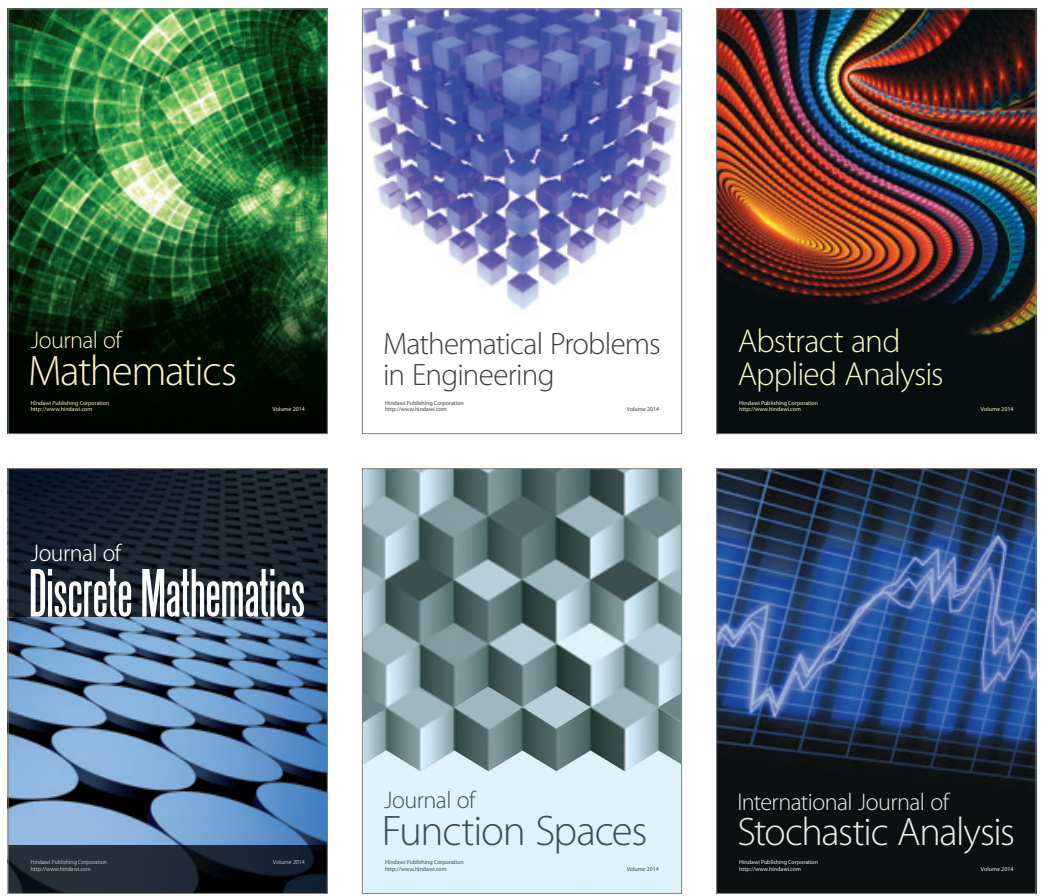

Journal of

Function Spaces

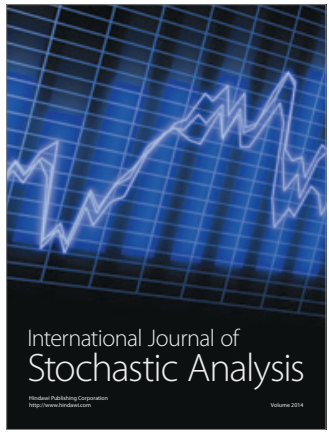

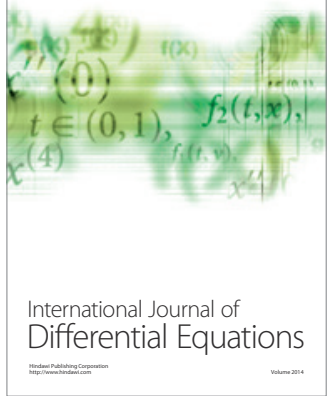
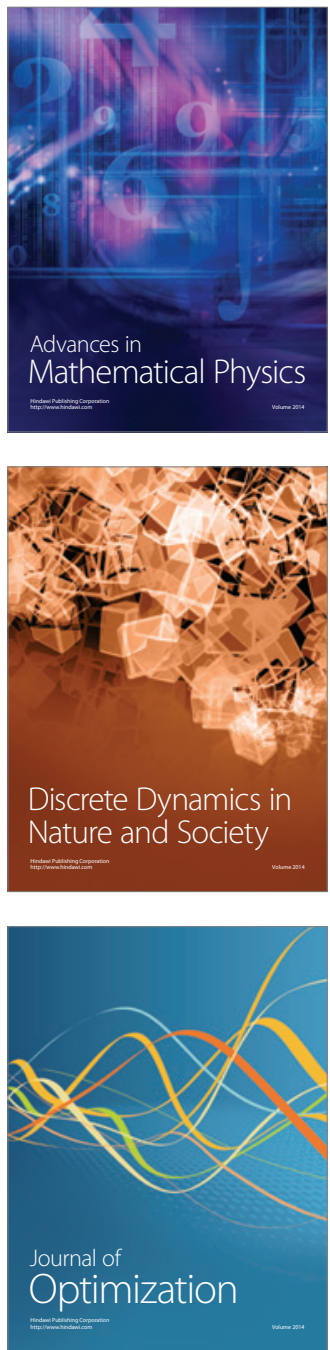